\title{
Rheumatoid arthritis in a tribal Xhosa the Transkei, Southern Africa
}

\author{
O. L. MEYERS, ${ }^{*}$ G. DAYNES, $\uparrow$ AND P. BEIGHTON +
}

From Groote Schuur Hospital and Department of Medicine, University of Cape Town Medical School,** St. Lucy's Hospital, Tsolo, Transkei: $†$ and Department of Human Genetics, University of Cape Town Medical School, $\ddagger$ South Africa

SUMMARY An epidemiological survey of rheumatoid arthritis (RA) was undertaken in a tribş Xhosa community in the Transkei of Southern Africa. 577 respondents aged 18 and over were examined clinically and of these, 549 were investigated radiologically and 482 serologically. Th\& presence of RA was then assessed by means of a modification of the Rome criteria, as used in previous comparable surveys.

The prevalence of 'definite' RA in the adults aged 18 and over in this population was $0.68 \%$ and of 'probable' RA, $1 \cdot 6 \%$. The combined 'definite' and 'probable' prevalence was $2.2 \%$. The relativel low prevalence of RA in this population is consistent with the results of other surveys in unsophist cated African Negro populations in West Africa and South Africa, and contrasts with the higher prevalence encountered in an urbanized South African Negro community and in populations i i Europe and the USA.

It is well established that there are considerable geographical variations in the prevalence of rheumatoid arthritis (RA) (Lawrence et al., 1966). Uncertainty remains as to whether these discrepancies are the result of ethnic or environmental factors. However, recent epidemiological studies in South Africa have indicated that although RA is very uncommon in African Negroes living in a rural environment, the prevalence in urbanized Africans approaches that normally encountered in Western Europe (Beighton et al., 1975; Solomon et al., 1975a).

If RA is indeed rare in the rural Negro population of this country, it is reasonable to suppose that the condition would be equally uncommon in less sophisticated tribal circumstances. In order to further elucidate this situation and to confirm the previous findings, an epidemiological investigation of RA was undertaken in a Xhosa community, living in a tribal environment in the Transkei.

\section{Population}

The survey concerned the inhabitants of 2 adjacent villages, Zinchuka and Esingeni, in the Tsolo district of the Transkei, South Africa. This Xhosa

Accepted for publication May 17, 1976

Correspondence to Prof. P. Beighton, Department of Human

Genetics, Medical School, Observatory 7900, South Africa population is descended from the Negroes o Central Africa and is closely related to other Bantig speaking groups such as the Zulu and the Tswana.

The community lived in tribal circumstances with an unsophisticated life-style, the cultivation of maize and cattle husbandry being the main occupa tions. As a large proportion of the males hac migrated to the cities to find employment, there was a preponderance of females in the population.

The villages have been served by St. Cuthbert? mission and St. Lucy's Hospital since the last cen tury, and the unstinted collaboration of the resporp dents in the survey was the result of this long-standing relationship. Demographic details have been given in a companion paper concerning serund uric acid concentrations in this tribal Xhosa popula tion (Beighton et al., 1976).

\section{Methods}

The investigation techniques were similar to those used in previous surveys in the South African series (Solomon et al., $1975 \mathrm{a}, \mathrm{b}$ ) and the description of the methodology of the present survey is therefore confined to a brief outline.

SURVEY METHODS

A preliminary census was carried out in randoml 
selected areas in the two villages to give information on the basic structure of the population and to provide a pool of potential respondents. During June and July 1973, using the facilities of St. Lucy's Hospital, the respondents were examined clinically, radiographs were obtained of their hands, and venepuncture was undertaken in all individuals aged 18 and over. Anteroposterior radiographs of the pelvis and hip joints, and lateral views of the lumbar spine were also obtained in a proportion of adults, aged 45 and over, for studies of other skeletal conditions.

In the concluding phase of the survey the patients were visited in their homes to ensure adequate completion rates. Completion rates were $96 \%$ for the clinical aspects, $91 \%$ for the radiographic studies, and $80 \%$ for the serological investigations.

\section{LABORATORY METHODS}

Sera were separated within 4 hours of collection, stored at $-20^{\circ} \mathrm{C}$, and transported by air in insulated containers to the Medical School, University of Cape Town. Rheumatoid factor (RF) in the serum was measured by two methods: the human latex fixation test (LFT) and the human erythrocyte agglutination test (HEAT). For the sake of conformity with previous investigations the LFT was carried out after prior heating of the serum to $56^{\circ} \mathrm{C}\left(\mathrm{LFT}^{56}\right)$, according to the modification introduced by Valkenburg (1963). An LFT ${ }^{56}$ titre of 1 in 640 or over and an HEAT titre of 1 in 32 or over was considered to be positive. All sera were also tested for RF by the standard Singer and Plotz method (Singer et al., 1962.)

EVALUATION OF RA BY COMBINED CRITERIA In the previous surveys in South Africa it became apparent that a clinical history was unreliable, owing to language barriers and to differing cultural concepts of disease processes. For this reason a modification of the 'Rome criteria' (Kellgren et al., $1963 \mathrm{a}, \mathrm{b})$ was used to assess the presence of RA. The following objective criteria were used.

(i) Clinically apparent symmetrical deformity of peripheral joints, especially of the metacarpophalangeal or metatarsophalangeal joints, with involvement of at least one hand or foot.

(ii) Radiographic changes of RA of grade 2 or more (de Graaf et al., 1963; Kellgren et al., 1963b).

(iii) Positive serological tests for RF.

Individuals fulfilling all 3 criteria were designated 'definite' RA, while those fulfilling 2 of the criteria were considered to have 'probable' RA. Diagnosis was negated by any of the exclusions listed in the American Rheumatism Association's criteria of 1958 (Ropes et al., 1959).

\section{Results}

\section{CLINICAL ASSESSMENT}

577 respondents aged 18 years and over were examined for objective evidence of inflammatory arthritis. This was considered to be present in 10 of them. 3 females, aged 50, 76, and 78 years, fulfilled the criteria for definite RA. Of these, one had severe crippling arthritis, advanced grade 4 erosive arthritis, and positive tests for RF. The other 2 women had a mild polyarthritis, grade 2 erosive arthritis, and positive tests for RF. The remaining 7 respondents who were considered to have an inflammatory arthritis all had very mild disease. None of these respondents showed any radiological evidence of RA but 2 had positive tests for RF (one had positive LFT and HEAT).

None of the 400 respondents aged 17 or under had any clinical evidence of polyarthritis. However, as their serological and radiographical status was not investigated, this group have been deliberately excluded from the polyarthritis aspect of the survey. No respondent had any clinical or radiographic evidence of ankylosing spondylitis.

\section{RADIOLOGICAL ASSESSMENT}

The radiographs of 549 respondents aged 18 or over were examined for evidence of erosive arthritis by one investigator (O.L.M.). There were 18 examples of grade 2 changes (14 females, 4 males) and one subject with advanced RA. In addition, a single pocketed lesion was present in 8 instances. These minor abnormalities were not accepted as evidence of erosive arthritis because of their consistent location on the medial side of the base of the third or fourth proximal phalanges. This uniformity of appearance and distribution suggested that some mechanism other than RA was operative.

Apart from the 3 subjects who were considered to have definite RA, none of the respondents with erosive changes had any clinical evidence of inflammatory arthritis. 9 of the respondents with grade 2 radiological changes also had positive tests for RF (LFT 9, HEAT 2).

\section{S ROLOGICAL ASSESSMENT}

The LFT $^{56}$ studies were performed on the sera of 482 respondents (77 males, 405 females), of whom 83 were found to have positive titres. This group contained 3 subjects with 'definite' RA, 2 with a mild clinical polyarthritis, and 9 with erosive arthritis. The remaining 71 respondents showed neither clinical nor radiological evidence of RA. The overall prevalence of positive LFTs in the survey population was $17 \%$, increasing with age from $7.2 \%$ in the 18-24 age cohort to $19 \cdot 7 \%$ in the $65-74$ age cohort. 
The HEAT test was performed on the sera of 476 respondents ( 76 males, 400 females), of whom 10 had positive titres. These HEAT-positive respondents comprised 2 subjects with definite RA and 8 with neither clinical nor radiological evidence of RA. The overall prevalence of HEAT-positive reactions was $2.1 \%$ and, as with the LFT, there was an increase in positive tests with age, ranging from $0 \%$ in the $18-24$ age cohort to $3.2 \%$ in the 64-75 age cohort.

All the sera were tested by the standard latex agglutination test of Singer and Plotz. 40 sera were proved positive by this method, and in 7 this technique gave positive results when all other tests were negative. This compares with 50 by the LFT $^{56}$ method. In 33 sera the 2 tests were concordant. All the sera of the 'definite' RA group were positive by the Singer and Plotz method.

EVALUATION BY THE COMBINED CRITERIA As clinical histories were considered to be unreliable, these were omitted from the overall assessment. A diagnosis of 'definite' RA was accepted if the remaining three Rome criteria were fulfilled, and 'probable' RA when two of the three criteria were met.

577 respondents over the age of 18 years were examined clinically, and of these, 549 were investigated radiologically and in $\mathbf{4 8 2}$ serological studies were undertaken. Adequate clinical, radiological, and serological data were finally available in 433 respondents and these were then analysed by the combined criteria.

Three respondents were considered to have 'definite' RA, representing a prevalence of $0.68 \%$ for individuals aged 18 and over. 7 respondents met two of the criteria for 'probable' RA, giving a prevalence of $1.6 \%$. The combined 'definite' and 'probable' RA prevalence was $2 \cdot 2 \%$.

\section{Discussion}

Clinically apparent inflammatory polyarthritis was present in $1.7 \%$ of 577 respondents aged 18 years and over. The stigmata of the disease were mild and with one exception none of the characteristic deformities of RA was encountered. This individual had the classical changes of RA, and was bedridden. These findings are in accordance with those of the previously mentioned study in the rural Tswana people of South Africa and with the reports concerning the mild clinical manifestations of RA in Negro populations in other regions of Africa (Hall, 1966; Greenwood, 1969; Gelfand, 1969; Anderson, 1971).

Erosive arthritis was observed in $3.4 \%$ of 549 hand radiographs. This prevalence is a little higher $\frac{\mathrm{D}}{\mathrm{N}}$ than figures generally quoted from other parts of the ? world. However, the changes were generally of $\overrightarrow{\vec{F}}$ minor degree and with one exception were grade $2-\frac{7}{0}$ or less. In 3 subjects the radiological features corre-c lated with clinical and serological evidence for RA, while in 7 subjects the radiological signs correlated $\mathbb{\Phi}$ with a positive RF test.

The LFT $^{56}$ was positive in $17 \%$ of the population, while the HEAT was positive in $2.1 \%$. Using the $\vec{O}$ conventional Singer and Plotz method, the sera from $\overrightarrow{\vec{\omega}}$ the Xhosa population gave a positive rate of $8.6 \%$. These high figures for LFT ${ }^{56}$ are similar to those@ previously reported from Nigeria and South? Africa (Muller, 1970; Solomon et al., 1975b). As in other populations the prevalence of positive RF $\overrightarrow{-}$ tests increased with aging.

The high prevalence of positive latex titres in the응 Xhosa population could result from infection with tuberculosis or syphilis, both of which produce ${ }_{\infty}^{T}$ positive latex titres, and which are common in the음 Transkei. It is noteworthy that malaria, which has been implicated as a causative factor in LFT positivity in other African Negro peoples (Greenwood, $\vec{\theta}$ 1968), is not endemic in the Transkei.

The low prevalence of RA in the Xhosa, assessed on a basis of the modified combined criteria, is similar to that in the rural Tswana, and is in keeping with the data concerning tribal peoples in Nigeria (Greenwood, 1969; Muller, 1970). The reason for the $\frac{\circ}{\mathbb{Q}}$ discrepancy in prevalence of RA in the urban and nonurban South African Negro groups is unknown. However, in view of the ethnic similarity of the two groups previously studied, genetic factors do not? seem to play a major role. It may be relevant that RA appears to be a relatively recent disease in Western Europe, and it could be postulated that unknown environmental factors are responsible for the 3 . variations in distribution of RA in Southern Africa.

Lifestyles are changing in this country. It is important that the problem of distribution of RA is elucidated while tribal and rural cultural groups still live alongside urbanized peoples of similar ethnic stock.

We are grateful to the Xhosa community for their willing participation, and to the Department of ${ }_{\sigma}^{\omega}$ Bantu Administration for their permission for the survey to be carried out. We are indebted to Mrs? Greta Beighton for typing the manuscript.

The investigation was supported by grants from? the South African Medical Research Council, the University of Cape Town Staff Research Fund, and the Arthritis Foundation of South Africa (Cape $\frac{?}{\mathbb{D}}$ Western Branch). 


\section{References}

Anderson, I. (1971). Rheumatoid arthritis in the Bantu. South African Medical Journal, 44, 1227-1229.

Beighton, P., Solomon, L., and Valkenburg, H. (1975). Rheumatoid arthritis in a rural South African Negro population. Annals of the Rheumatic Diseases, 34, 136-141.

Beighton, P., Daynes, G., and Soskolne, C. L. (1976). Serum uric acid concentrations in a Xhosa community in the Transkei of Southern Africa. Annals of the Rheumatic Diseases, 35, 77-80.

de Graaf, R., Laine, V., and Lawrence, J. S. (1963). The Epidemiology of Chronic Rheumatism, Vol. 1, pp. 228-238. Ed. by J. H. Kellgren, M. R. Jeffrey, and J. Ball. Blackwell, Oxford.

Gelfand, M. (1969). Medical arthritis in African practice. Central African Journal of Medicine, 15, 131-135.

Greenwood, B. M. (1968). Autoimmune disease and parasitic infections in Nigerians. Lancet, 2, 380-382.

Greenwood, B. M. (1969). Polyarthritis in Western Nigeria. I. Rheumatoid arthritis. Annals of the Rheumatic Diseases, 28, 488-496.

Hall, L. (1966). Polyarthritis in Kenya. East African Medical Journal, 43, 161-170.

Kellgren, J. H., Jeffrey, M. R., and Ball, J. (1963a). The Epidemiology of Chronic Rheumatism, Vol. 1, p. 324. Blackwell, Oxford.

Kellgren, J. H., Jeffrey, M. R., and Ball, J. (1963b). The
Epidemiology of Chronic Rheumatism, Vol. 2. Blackwell, Oxford.

Lawrence, J. S., Behrend, T., Bennett, P. H., Bremner, J. M., Burch, T. A., Gofton, J., O'Brien, W., and Robinson, $\mathbf{H}$. (1966). Geographical studies on rheumatoid arthritis. Annals of the Rheumatic Diseases, 35, 425-432.

Muller, A. S. (1970). Population studies on the prevalence of rheumatic diseases in Liberia and Nigeria. M.D. thesis, University of Leiden.

Ropes, M. W., Bennett, G. A., Cobb, S., Jacox, R., and Jessar, R. A. (1959). Diagnostic criteria for rheumatoid factor. 1958 revision. Annals of the Rheumatic Diseases, 18, 49-53.

Singer, J. M., Plotz, C. M., Peralta, M., and Lyons, H. C. (1962). The presence of anti-gamma globulin factors in sera of patients with active pulmonary tuberculosis. Annals of Internal Medicine, 56, 545-552.

Solomon, L., Robin, G., and Valkenburg, H. A. (1975a). Rheumatoid arthritis in an urban South African Negro population. Annals of the Rheumatic Diseases, 34, 128-135.

Solomon, L., Beighton, P., Valkenburg, H. A., Robin, G., and Soskolne, C. L. (1975b). Rheumatic disorders in the South African Negro. I. Rheumatoid arthritis and ankylosing spondylitis. South African Medical Journal, 49, 1292-1296.

Valkenburg, H. A. (1963). Rheumatoid factor in populations. The Epidemiology of Chronic Rheumatism, Vol. 1, p. 74. Ed. by J. H. Kellgren, M. R. Jeffrey, and J. Ball. Blackwell, Oxford. 